\title{
Adaptive Semidiscrete Finite Element Methods for Semilinear Parabolic Integrodifferential Optimal Control Problem with Control Constraint
}

\author{
Zuliang Lu ${ }^{1,2}$ \\ ${ }^{1}$ School of Mathematics and Statistics, Chongqing Three Gorges University, Chongqing 404000, China \\ ${ }^{2}$ College of Civil Engineering and Mechanics, Xiangtan University, Xiangtan 411105, China
}

Correspondence should be addressed to Zuliang Lu; zulianglux@126.com

Received 27 April 2013; Revised 5 August 2013; Accepted 27 August 2013

Academic Editor: Dewei Li

Copyright (C) 2013 Zuliang Lu. This is an open access article distributed under the Creative Commons Attribution License, which permits unrestricted use, distribution, and reproduction in any medium, provided the original work is properly cited.

\begin{abstract}
The aim of this work is to study the semidiscrete finite element discretization for a class of semilinear parabolic integrodifferential optimal control problems. We derive a posteriori error estimates in $L^{2}\left(J ; L^{2}(\Omega)\right)$-norm and $L^{2}\left(J ; H^{1}(\Omega)\right)$-norm for both the control and coupled state approximations. Such estimates can be used to construct reliable adaptive finite element approximation for semilinear parabolic integrodifferential optimal control problem. Furthermore, we introduce an adaptive algorithm to guide the mesh refinement. Finally, a numerical example is given to demonstrate the theoretical results.
\end{abstract}

\section{Introduction}

With the advances of scientific computing, optimal control problems are now widely used in multidisciplinary applications such as physical, biological, medicine, engineering design, finance, fluid mechanics, and socioeconomic systems. As a result, more and more people will benefit greatly by learning to solve the optimal control problems numerically. Realizing such growing needs, books and papers on optimal control put more weight on numerical methods.

In modeling a wide range of problems for physical, economic, and social processes, optimal control problems described by integrodifferential equations play an important role. Parabolic integrodifferential optimal control problems are very important model in engineering numerical simulation, for example, biology mechanics, nuclear reaction dynamics, heat conduction in materials with memory, viscoelasticity, and so forth. Finite element approximation of optimal control problems has a very important status in the numerical methods for these problems. The finite element approximation of optimal control problem by piecewise constant functions was well investigated by Falk [1] and Geveci [2]. The finite element methods for semilinear elliptic optimal control problems were discussed by Casas et al. in [3]. In [4], the author studied the finite element discretization for a class of quadratic boundary optimal control problems governed by nonlinear elliptic equations and obtained a posteriori error estimates for the coupled state and control approximation. Many introductions about the numerical computation method for optimal control problems can be found in [5-8].

As one of important kinds of optimal control problems, parabolic integrodifferential optimal control problem is widely used in scientific and engineering computing. The literature in this aspect was huge, see; for example, [9]. In [10], Brunner and Yan analyzed finite element Galerkin discretization for a class of optimal control problems governed by integral equations and integrodifferential equations and derived a priori error estimates and a posteriori error estimators for the approximation solutions.

Adaptive finite element method is the most important method to boost accuracy of the finite element discretization. It ensures a higher density of nodes in certain area of the given domain, where the solution is discontinuous or more difficult to approximate, using an a posteriori error indicator. A posteriori error estimates are computable quantities in terms of the discrete solution and they measure the actual discrete errors without the knowledge of exact solutions. They are essential in designing algorithms for mesh which 
equidistribute the computational effort and optimize the computation. The literature in this was huge. In [11], the authors presented an a posteriori error analysis for finite element approximation of distributed convex elliptic optimal control problems. They derived a posteriori error estimates for the coupled state and control approximations. Such estimates can be used to construct reliable adaptive finite element approximation schemes for control problems. In [12], Verfürth gave a general framework for deriving a posteriori error estimates for approximate solutions of nonlinear elliptic equations. He obtained a posteriori error estimates, which can easily be computed from the given data of the problem and the computed numerical solution and which give global upper and local lower bounds on the error of the numerical solution. Some of techniques directly relevant to our work can be found in [11, 12]. Recently, in [13-16], we derived a priori error estimates and a posteriori error estimates for optimal control problems using mixed finite element methods. Although a posteriori error estimates of finite element approximation were widely used in numerical simulations, they have not yet been utilized in nonlinear parabolic integrodifferential optimal control problem.

In this paper, we adopt the standard notation $W^{m, p}(\Omega)$ for Sobolev spaces on $\Omega$ with a norm $\|\cdot\|_{m, p}$ given by $\|v\|_{m, p}^{p}=\sum_{|\alpha| \leq m}\left\|D^{\alpha} v\right\|_{L^{p}(\Omega)}^{p}$ and a seminorm $|\cdot|_{m, p}$ given by $|v|_{m, p}^{p}=\sum_{|\alpha|=m}\left\|D^{\alpha} v\right\|_{L^{p}(\Omega)}^{p}$. We set $W_{0}^{m, p}(\Omega)=\{v \in$ $\left.W^{m, p}(\Omega):\left.v\right|_{\partial \Omega}=0\right\}$. For $p=2$, we denote $H^{m}(\Omega)=$ $W^{m, 2}(\Omega), H_{0}^{m}(\Omega)=W_{0}^{m, 2}(\Omega)$, and $\|\cdot\|_{m}=\|\cdot\|_{m, 2},\|\cdot\|=$ $\|\cdot\|_{0,2}$. We denote by $L^{s}\left(0, T ; W^{m, p}(\Omega)\right)$ the Banach space of all $L^{s}$ integrable functions from $J$ to $W^{m, p}(\Omega)$ with norm $\|v\|_{L^{s}\left(J ; W^{m, p}(\Omega)\right)}=\left(\int_{0}^{T}\|v\|_{W^{m, p}(\Omega)}^{s} d t\right)^{1 / s}$ for $s \in[1, \infty)$, and the standard modification for $s=\infty$. The details can be found in [9].

In this paper, we derive a posteriori error estimates for a class of semilinear parabolic integrodifferential optimal control problems. To the best of our knowledge in the context of semilinear parabolic integrodifferential optimal control problems, these estimates are new. We consider the following semilinear parabolic integrodifferential optimal control problems:

$$
\min _{u(t) \in K \subset U}\left\{\int_{0}^{T}\left(\frac{1}{2}\left\|y-y_{0}\right\|^{2}+\frac{\alpha}{2}\|u\|^{2}\right) d t\right\}
$$

subject to the state equation

$$
\begin{gathered}
y_{t}-\operatorname{div}(A \nabla y(x, t)) \\
-\int_{0}^{t} \operatorname{div}(\psi(t, \tau) \nabla y(x, \tau)) d \tau+\phi(y) \\
=f+B u, \quad x \in \Omega, t \in J, \\
y(x, t)=0, \quad x \in \partial \Omega, t \in J, \\
y(x, 0)=y_{0}(x), \quad x \in \Omega,
\end{gathered}
$$

where the bounded open set $\Omega \subset \mathbb{R}^{2}$ is 2 regular convex polygon with boundary $\partial \Omega, J=(0, T], f \in L^{2}(\Omega), \psi=$ $\psi(x, t, \tau)=\psi_{i, j}(x, t, \tau)_{2 \times 2} \in C^{\infty}\left(0, T ; L^{2}(\bar{\Omega})\right)^{2 \times 2}, y_{0} \in H^{1}(\Omega)$, $\alpha$ is a positive constant, and $B$ is a continuous linear operator from $K$ to $L^{2}(\Omega)$. For any $R>0$, the function $\phi(\cdot) \epsilon$ $W^{2, \infty}(-R, R), \phi^{\prime}(y) \in L^{2}(\Omega)$ for any $y \in L^{2}\left(J ; H_{0}^{1}(\Omega)\right)$, and $\phi^{\prime}(y) \geq 0$. We assume that the coefficient matrix $A(x)=$ $\left(a_{i, j}(x)\right)_{2 \times 2} \in\left(W^{1, \infty}(\Omega)\right)^{2 \times 2}$ is a symmetric positive definite matrix and there is a constant $c>0$ satisfying for any vector $\mathbf{X} \in \mathbb{R}^{2}, \mathbf{X}^{t} A \mathbf{X} \geq c\|\mathbf{X}\|_{\mathbb{R}^{2}}^{2}$. Here, $K$ denotes the admissible set of the control variable defined by

$$
K=\left\{u(x, t) \in U=L^{2}\left(J ; L^{2}(\Omega)\right): u(x, t) \geq 0, t \in J\right\} .
$$

Assume that there are constants $c$ and $C$, such that for all $t$ and $\tau$ in $[0, T]$ :

$$
\begin{gathered}
a(z, z) \geq c\|z\|_{1, \Omega}^{2}, \quad \forall z \in V, \\
|a(z, w)| \leq C\|z\|_{1, \Omega} \cdot\|w\|_{1, \Omega}, \quad \forall z, w \in V, \\
|\psi(t, \tau ; z, w)| \leq C\|z\|_{1, \Omega} \cdot\|w\|_{1, \Omega}, \quad \forall z, w \in V .
\end{gathered}
$$

The plan of this paper is as follows. In the next section, we present the finite element discretization for semilinear parabolic integrodifferential optimal control problems. A posteriori error estimates are established for the optimal control problems in Section 3. In Section 4, we introduce an adaptive algorithm to guide the mesh refinement. In Section 5, a numerical example is given to demonstrate our theoretical results. Finally, we analyze the conclusion and future work in Section 6.

\section{Finite Elements for Integrodifferential Optimal Control}

We will now describe the finite element discretization of semilinear parabolic integrodifferential optimal control problems (1)-(2). Let $V=H_{0}^{1}(\Omega)$ and $W=L^{2}(\Omega)$. Let

$$
\begin{gathered}
a(y, w)=\int_{\Omega}(A \nabla y) \cdot \nabla w, \quad \forall y, w \in V, \\
\psi(t, \tau ; z, w)=(\psi(t, \tau) \nabla z, \nabla w), \quad \forall z, w \in V \times V, \\
(u, v)=\int_{\Omega} u v, \quad \forall(u, v) \in W \times W, \\
\left(f_{1}, f_{2}\right)=\int_{\Omega} f_{1} f_{2}, \quad \forall\left(f_{1}, f_{2}\right) \in W \times W .
\end{gathered}
$$

Then, the semilinear parabolic integrodifferential optimal control problems (1)-(2) can be restated as

$$
\min _{u(t) \in K}\left\{\int_{0}^{T}\left(\frac{1}{2}\left\|y-y_{0}\right\|^{2}+\frac{\alpha}{2}\|u\|^{2}\right) d t\right\}
$$


subject to

$$
\begin{gathered}
\left(y_{t}, w\right)+a(y, w)+\int_{0}^{t} \psi(t, \tau ; y(\tau), w) d \tau+(\phi(y), w) \\
=(f+B u, w), \quad \forall w \in V, t \in J, \\
y(x, 0)=y_{0}(x), \quad x \in \Omega,
\end{gathered}
$$

where the inner product in $L^{2}(\Omega)$ or $L^{2}(\Omega)^{2}$ is indicated by $(\cdot, \cdot)$. From Yanik and Fairweather [17], we know that the above weak form has at least one solution in $y \in W(0, T)=$ $\left\{w \in L^{2}\left(0, T ; H^{1}(\Omega)\right), w_{t}^{\prime} \in L^{2}\left(0, T ; H^{-1}(\Omega)\right)\right\}$.

It is well known (see, e.g., [11]) that the optimal control problem has a solution $(y, u)$, and if a pair $(y, u)$ is the solution of (8)-(9), then there is a costate $p \in V$ such that triplet $(y, p, u)$ satisfies the following optimality conditions:

$$
\begin{gathered}
\left(y_{t}, w\right)+a(y, w) \\
+\int_{0}^{t} \psi(t, \tau ; y(\tau), w) d \tau+(\phi(y), w) \\
=(f+B u, w), \quad \forall w \in V, \\
y(x, 0)=y_{0}(x), \quad x \in \Omega, \\
-\left(p_{t}, w\right)+a(q, p)+\int_{t}^{T} \psi(\tau, t ; q, p(\tau)) d \tau+\left(\phi^{\prime}(y) p, q\right) \\
=\left(y-y_{0}, q\right), \quad \forall q \in V, \\
p(x, T)=0, \quad x \in \Omega, \\
\int_{0}^{T}\left(\alpha u+B^{*} p, v-u\right)_{U} d t \geq 0, \quad \forall v \in K,
\end{gathered}
$$

where $B^{*}$ is the adjoint operator of $B$. In the rest of the paper, we will simply write the product as $(\cdot, \cdot)$ whenever no confusion should be caused.

Let us consider the finite element approximation of the optimal control problem (8)-(9). Again here we consider only $n$-simplex elements and conforming finite elements.

Let $\mathscr{T}^{h}$ be regular partition of $\Omega$. Associated with $\mathscr{T}^{h}$ is a finite dimensional subspace $V_{h}$ of $C(\bar{\Omega})$, such that $\left.\chi\right|_{\tau}$ are polynomials of $m$-order $(m \geq 1)$ for all $\chi \in V_{h}$ and $\tau \in \mathscr{T}^{h}$. It is easy to see that $V_{h} \subset V$. Let $h_{\tau}$ denote the maximum diameter of the element $\tau$ in $\mathscr{T}^{h}, h=\max _{\tau \in \mathscr{T}^{h}}\left\{h_{\tau}\right\}$.

Due to the limited regularity of the optimal control $u$ in general, there will be no advantage in considering higherorder finite element spaces for the control. So, we only consider the piecewise constant finite element space for the approximation of the control, though higher-order finite element spaces will be used to approximate the state and the costate. Let $P_{0}(\tau)$ denote all the 0 -order polynomial over $\tau$. Therefore, we take $K_{h}=\left\{u \in K:\left.u(x, t)\right|_{\tau} \in\right.$ $\left.P_{0}(\tau)\right\}$. In addition, $C$ or $c$ denotes a general positive constant independent of $h$.
By the definition of finite element subspace, the finite element discretization of (8)-(9) is as follows: compute $\left(y_{h}, u_{h}\right) \in V_{h} \times K_{h}$ such that

$$
\begin{gathered}
\min _{u_{h} \in K_{h}}\left\{\int_{0}^{T}\left(\frac{1}{2}\left\|y_{h}-y_{0}\right\|^{2}+\frac{\alpha}{2}\left\|u_{h}\right\|^{2}\right) d t\right\} \\
\left(y_{h t}, w_{h}\right)+a\left(y_{h}, w_{h}\right)+\int_{0}^{t} \psi\left(t, \tau ; y_{h}(\tau), w_{h}\right) d \tau \\
+\left(\phi\left(y_{h}\right), w_{h}\right)=\left(f+B u_{h}, w_{h}\right), \\
y_{h}(x, 0)=y_{0}^{h}(x), \quad x \in \Omega,
\end{gathered}
$$

where $w_{h} \in V_{h}, y_{0}^{h} \in V_{h}$ is an approximation of $y_{0}$.

Again, it follows that the optimal control problems (15)(17) have a solution $\left(y_{h}, u_{h}\right)$, and if a pair $\left(y_{h}, u_{h}\right)$ is the solution of (15)-(17), then there is a costate $p_{h} \in V_{h}$ such that triplet $\left(y_{h}, p_{h}, u_{h}\right)$ satisfies the following optimality conditions:

$$
\begin{gathered}
\left(y_{h t}, w_{h}\right)+a\left(y_{h}, w_{h}\right)+\int_{0}^{t} \psi\left(t, \tau ; y_{h}(\tau), w_{h}\right) d \tau \\
+\left(\phi\left(y_{h}\right), w_{h}\right)=\left(f+B u_{h}, w_{h}\right), \\
y_{h}(x, 0)=y_{0}^{h}(x), \quad x \in \Omega, \\
-\left(p_{h t}, w_{h}\right)+a\left(q_{h}, p_{h}\right)+\int_{t}^{T} \psi\left(\tau, t ; q_{h}, p_{h}(\tau)\right) d \tau \\
+\left(\phi^{\prime}\left(y_{h}\right) p_{h}, q_{h}\right)=\left(y_{h}-y_{0}, q_{h}\right), \\
p_{h}(x, T)=0, \quad x \in \Omega, \\
\int_{0}^{T}\left(\alpha u_{h}+B^{*} p_{h}, v_{h}-u_{h}\right) d t \geq 0,
\end{gathered}
$$

where $w_{h}, q_{h} \in V_{h}$, and $v_{h} \in K_{h}$.

In the rest of the paper, we will use some intermediate variables. For any control function $u_{h} \in K$, we first define the state solution $\left(y\left(u_{h}\right), p\left(u_{h}\right)\right)$ satisfying

$$
\begin{aligned}
& \left(y_{t}\left(u_{h}\right), w\right)+a\left(y\left(u_{h}\right), w\right) \\
& \quad+\int_{0}^{t} \psi\left(t, \tau ; y\left(u_{h}\right)(\tau), w\right) d \tau \\
& \quad+\left(\phi\left(y\left(u_{h}\right)\right), w\right)=\left(f+B u_{h}, w\right),
\end{aligned}
$$

$\forall w \in V, \quad y\left(u_{h}\right)(x, 0)=y_{0}(x), \quad x \in \Omega$,

$$
\begin{aligned}
& -\left(p_{t}\left(u_{h}\right), q\right)+a\left(q, p\left(u_{h}\right)\right)+\int_{t}^{T} \psi\left(\tau, t ; q, p\left(u_{h}\right)(\tau)\right) d \tau \\
& +\left(\phi^{\prime}\left(y\left(u_{h}\right)\right) p\left(u_{h}\right), q\right)
\end{aligned}
$$

$$
=\left(y\left(u_{h}\right)-y_{0}, q\right), \quad \forall q \in V, \quad p\left(u_{h}\right)(x, T)=0, \quad x \in \Omega \text {. }
$$
[9].
Now, we restate the following well-known estimates in 
Lemma 1. Let $\widehat{\pi}_{h}$ be the Clément-type interpolation operator defined in [9]. Then for any $v \in H^{1}(\Omega)$ and all element $\tau$,

$$
\begin{gathered}
\left\|v-\widehat{\pi}_{h} v\right\|_{L^{2}(\tau)}+h_{\tau}\left\|\nabla\left(v-\widehat{\pi}_{h} v\right)\right\|_{L^{2}(\tau)} \\
\leq C h_{\tau} \sum_{\bar{\tau}^{\prime} \cap \bar{\tau} \neq \emptyset}|v|_{L^{2}\left(\tau^{\prime}\right)}, \\
\left\|v-\widehat{\pi}_{h} v\right\|_{L^{2}(l)} \leq C h_{l}^{1 / 2} \sum_{l \subset \bar{\tau}^{\prime}}|\nabla v|_{L^{2}\left(\tau^{\prime}\right)},
\end{gathered}
$$

where $l$ is the edge of the element.

For $\varphi \in W_{h}$, we will write

$$
\begin{aligned}
\phi(\varphi)-\phi(\rho) & =-\widetilde{\phi}^{\prime}(\varphi)(\rho-\varphi) \\
& =-\phi^{\prime}(\rho)(\rho-\varphi)+\widetilde{\phi}^{\prime \prime}(\varphi)(\rho-\varphi)^{2},
\end{aligned}
$$

where

$$
\begin{gathered}
\widetilde{\phi}^{\prime}(\varphi)=\int_{0}^{1} \phi^{\prime}(\varphi+s(\rho-\varphi)) d s, \\
\widetilde{\phi}^{\prime \prime}(\varphi)=\int_{0}^{1}(1-s) \phi^{\prime \prime}(\rho+s(\varphi-\rho)) d s
\end{gathered}
$$

are bounded functions in $\bar{\Omega}[12]$.

\section{A Posteriori Error Estimates}

In this section, we will obtain a posteriori error estimates for semilinear parabolic integrodifferential optimal control problems. Firstly, we estimate the error $\left\|u-u_{h}\right\|_{L^{2}\left(J ; L^{2}(\Omega)\right)}$.

For given $u \in K$, let $M$ be the inverse operator of the state equation (10), such that $y(u)=M B u$ is the solution of the state equation (10). Similarly, for given $u_{h} \in K_{h}, y_{h}\left(u_{h}\right)=$ $M_{h} B u_{h}$ is the solution of the discrete state equation (16). Let

$$
\begin{gathered}
S(u)=\frac{1}{2}\left\|M B u-y_{0}\right\|^{2}+\frac{\alpha}{2}\|u\|^{2}, \\
S_{h}\left(u_{h}\right)=\frac{1}{2}\left\|M_{h} B u_{h}-y_{0}\right\|^{2}+\frac{\alpha}{2}\left\|u_{h}\right\|^{2} .
\end{gathered}
$$

It is clear that $S$ and $S_{h}$ are well defined and continuous on $K$ and $K_{h}$. Also the functional $S_{h}$ can be naturally extended on $K$. Then (8) and (15) can be represented as

$$
\begin{gathered}
\min _{u \in K}\{S(u)\}, \\
\min _{u_{h} \in K_{h}}\left\{S_{h}\left(u_{h}\right)\right\} .
\end{gathered}
$$

It can be shown that

$$
\begin{gathered}
\left(S^{\prime}(u), v\right)=\left(\alpha u+B^{*} p, v\right), \\
\left(S^{\prime}\left(u_{h}\right), v\right)=\left(\alpha u_{h}+B^{*} p\left(u_{h}\right), v\right), \\
\left(S_{h}^{\prime}\left(u_{h}\right), v\right)=\left(\alpha u_{h}+B^{*} p_{h}, v\right),
\end{gathered}
$$

where $p\left(u_{h}\right)$ is the solution of (23)-(25).
In many applications, $S(\cdot)$ is uniformly convex near the solution $u$ (see, e.g., [18]). The convexity of $S(\cdot)$ is closely related to the second-order sufficient conditions of the control problems, which was assumed in many studies on numerical methods of the problems. If $S(\cdot)$ is uniformly convex, then there is a $c>0$, such that

$$
\int_{0}^{T}\left(S^{\prime}(u)-S^{\prime}\left(u_{h}\right), u-u_{h}\right) d t \geq c\left\|u-u_{h}\right\|_{L^{2}\left(J ; L^{2}(\Omega)\right)}^{2},
$$

where $u$ and $u_{h}$ are the solutions of (31) and (32), respectively. We will assume the above inequality throughout this paper.

Let $p\left(u_{h}\right)$ be the solution of $(23)-(25)$; we establish the following error estimate.

Theorem 2. Let $u$ and $u_{h}$ be the solutions of (31) and (32), respectively. Assume that $K_{h} \subset K$. In addition, assume that $\left.\left(S_{h}^{\prime}\left(u_{h}\right)\right)\right|_{\tau} \in H^{s}(\tau)$, for all $\tau \in \mathscr{T}_{h},(s=0,1)$, and there is a $v_{h} \in K_{h}$ such that

$$
\left|\left(S_{h}^{\prime}\left(u_{h}\right), v_{h}-u\right)\right| \leq C \sum_{\tau \in \mathscr{T}_{h}} h_{\tau}\left\|S_{h}^{\prime}\left(u_{h}\right)\right\|_{H^{s}(\tau)}\left\|u-u_{h}\right\|_{L^{2}(\tau)}^{s} .
$$

Then, one has

$$
\left\|u-u_{h}\right\|_{L^{2}\left(J ; L^{2}(\Omega)\right)}^{2} \leq C \eta_{1}^{2}+C\left\|p_{h}-p\left(u_{h}\right)\right\|_{L^{2}\left(J ; H^{1}(\Omega)\right)}^{2},
$$

where

$$
\eta_{1}^{2}=\int_{0}^{T} \sum_{\tau \in \mathscr{T}_{h}} h_{\tau}^{1+s}\left\|\alpha u_{h}+B^{*} p_{h}\right\|_{H^{s}(\tau)}^{1+s} d t
$$

Proof. It follows from (31) and (32) that

$$
\begin{gathered}
\int_{0}^{T}\left(S^{\prime}(u), u-v\right) \leq 0, \quad \forall v \in K, \\
\int_{0}^{T}\left(S_{h}^{\prime}\left(u_{h}\right), u_{h}-v_{h}\right) \leq 0, \quad \forall v_{h} \in K_{h} \subset K .
\end{gathered}
$$

Then it follows from (35), (39), and the Schwartz inequality, that

$$
\begin{aligned}
c \| u- & u_{h} \|_{L^{2}\left(J ; L^{2}(\Omega)\right)}^{2} \\
& \leq \int_{0}^{T}\left(S^{\prime}(u)-S^{\prime}\left(u_{h}\right), u-u_{h}\right) d t \\
& \leq-\int_{0}^{T}\left(S^{\prime}\left(u_{h}\right), u-u_{h}\right) d t \\
= & \int_{0}^{T}\left\{\left(S_{h}^{\prime}\left(u_{h}\right), u_{h}-u\right)\right. \\
& \left.\quad+\left(S_{h}^{\prime}\left(u_{h}\right)-S^{\prime}\left(u_{h}\right), u-u_{h}\right)\right\} d t
\end{aligned}
$$




$$
\begin{aligned}
& \leq \int_{0}^{T}\left\{\left(S_{h}^{\prime}\left(u_{h}\right), v_{h}-u\right)\right. \\
& \left.+\left(S_{h}^{\prime}\left(u_{h}\right)-S^{\prime}\left(u_{h}\right), u-u_{h}\right)\right\} d t \\
& \leq C \int_{0}^{T}\left\{\sum_{\tau \in \mathscr{T}_{h}} h_{\tau}^{1+s}\left\|S_{h}^{\prime}\left(u_{h}\right)\right\|_{H^{s}(\tau)}^{1+s}\right. \\
& \left.\quad+\left\|S_{h}^{\prime}\left(u_{h}\right)-S^{\prime}\left(u_{h}\right)\right\|_{L^{2}(\Omega)}^{2}\right\} d t \\
& +\frac{c}{2}\left\|u-u_{h}\right\|_{L^{2}\left(J ; L^{2}(\Omega)\right)}^{2}
\end{aligned}
$$

It is not difficult to show that

$$
S_{h}^{\prime}\left(u_{h}\right)=\alpha u_{h}+B^{*} p_{h}, \quad S^{\prime}\left(u_{h}\right)=\alpha u_{h}+B^{*} p\left(u_{h}\right),
$$

where $p\left(u_{h}\right)$ is defined in (23)-(26). Thanks to (11), it is easy to derive

$$
\begin{aligned}
\int_{0}^{T} & \left\|S_{h}^{\prime}\left(u_{h}\right)-S^{\prime}\left(u_{h}\right)\right\|_{L^{2}(\Omega)} d t \\
& =\int_{0}^{T}\left\|B^{*}\left(p_{h}-p\left(u_{h}\right)\right)\right\|_{L^{2}(\Omega)} d t \\
& \leq C\left\|p_{h}-p\left(u_{h}\right)\right\|_{L^{2}\left(J ; L^{2}(\Omega)\right)} \\
& \leq C\left\|p_{h}-p\left(u_{h}\right)\right\|_{L^{2}\left(J ; H^{1}(\Omega)\right)} .
\end{aligned}
$$

Then, by the estimates (40) and (42), we can prove the requested result (36).

Now, we estimate the error $\left\|y\left(u_{h}\right)-y_{h}\right\|_{L^{2}\left(j ; H^{1}(\Omega)\right)}$.

Theorem 3. Let $\left(y\left(u_{h}\right), p\left(u_{h}\right)\right)$ and $\left(y_{h}, p_{h}\right)$ be the solutions of (23)-(26) and (18)-(22), respectively. Then

$$
\left\|y\left(u_{h}\right)-y_{h}\right\|_{L^{2}\left(J ; H^{1}(\Omega)\right)}^{2} \leq C \sum_{i=2}^{4} \eta_{i}^{2}
$$

where

$$
\begin{aligned}
\eta_{2}^{2}=\int_{0}^{T} \sum_{\tau \in \mathscr{T}^{h}} h_{\tau}^{2} \int_{\tau}( & f+B u_{h}-y_{h t}+\operatorname{div}\left(A \nabla y_{h}\right) \\
& +\int_{0}^{t} \operatorname{div}\left(\psi(t, \tau) \nabla y_{h}(\tau)\right) d \tau \\
& \left.-\phi\left(y_{h}\right)\right)^{2} d t
\end{aligned}
$$

$$
\begin{aligned}
\eta_{3}^{2}=\int_{0}^{T} \sum_{\tau \in \mathscr{T}^{h}} h_{l} \int_{\partial \tau} & {\left[\left(A \nabla y_{h}\right) \cdot n\right.} \\
& \left.+\int_{0}^{t}\left(\psi(t, \tau) \nabla y_{h}(\tau)\right) \cdot n d \tau\right]^{2} d l d t, \\
\eta_{4}^{2}= & \left\|y_{h}(x, 0)-y_{0}(x)\right\|_{L^{2}(\Omega)}^{2},
\end{aligned}
$$

where $l$ is a face of an element $\tau, h_{l}$ is the size of face $l$, and $\left[\left(A \nabla y_{h}\right) \cdot n\right]$ is the A-normal derivative jump over the interior face $l$, defined by

$$
\left[\left(A \nabla y_{h}\right) \cdot n\right]_{l}=\left(\left.A \nabla y_{h}\right|_{\tau_{l}^{1}}-\left.A \nabla y_{h}\right|_{\tau_{l}^{2}}\right) \cdot n,
$$

where $n$ is the unit normal vector on $l=\bar{\tau}_{l}^{1} \cap \bar{\tau}_{l}^{2}$ outwards $\tau_{l}^{1}$. For convenience, one defines $\left[\left(A \nabla y_{h}\right) \cdot n\right]_{l}=0$ when $l \subset \partial \Omega$.

Proof. Let $e_{I}^{y}$ be the Clément-type interpolator of $e^{y}$ defined in Lemma 1. It follows from (18) and (23) that we have

$$
\begin{aligned}
& \left(\left(y_{h}-y\left(u_{h}\right)\right)_{t}, w_{h}\right)+a\left(y_{h}-y\left(u_{h}\right), w_{h}\right) \\
& \quad+\int_{0}^{t} \psi\left(t, \tau ;\left(y_{h}-y\left(u_{h}\right)\right)(\tau), w_{h}\right) d \tau \\
& \quad+\left(\phi\left(y_{h}\right)-\phi\left(y\left(u_{h}\right)\right), w_{h}\right)=0, \quad \forall w_{h} \in V_{h} .
\end{aligned}
$$

Let $e^{y}=y_{h}-y\left(u_{h}\right)$; by using (46), then we obtain

$$
\begin{aligned}
& \left(\left(y_{h}-y\left(u_{h}\right)\right)_{t}, e^{y}\right)+a\left(y_{h}-y\left(u_{h}\right), e^{y}\right) \\
& \quad+\int_{0}^{t} \psi\left(t, \tau ;\left(y_{h}-y\left(u_{h}\right)\right)(\tau), e^{y}\right) d \tau \\
& \quad+\left(\phi\left(y_{h}\right)-\phi\left(y\left(u_{h}\right)\right), e^{y}\right) \\
& =\left(\left(y_{h}-y\left(u_{h}\right)\right)_{t}, e^{y}-e_{I}^{y}\right)+a\left(y_{h}-y\left(u_{h}\right), e^{y}-e_{I}^{y}\right) \\
& \quad+\int_{0}^{t} \psi\left(t, \tau ;\left(y_{h}-y\left(u_{h}\right)\right)(\tau), e^{y}-e_{I}^{y}\right) d \tau \\
& +\left(\phi\left(y_{h}\right)-\phi\left(y\left(u_{h}\right)\right), e^{y}-e_{I}^{y}\right) \\
& =\left(y_{h t}, e^{y}-e_{I}^{y}\right)+a\left(y_{h}, e^{y}-e_{I}^{y}\right) \\
& \quad+\int_{0}^{t} \psi\left(t, \tau ; y_{h}(\tau), e^{y}-e_{I}^{y}\right) d \tau \\
& \quad+\left(\phi\left(y_{h}\right), e^{y}-e_{I}^{y}\right)-\left(f+B u_{h}, e^{y}-e_{I}^{y}\right) \\
& =\sum_{\tau} \int_{\tau}\left(y_{h t}-\operatorname{div}\left(A \nabla y_{h}\right)\right. \\
& \quad-\int_{0}^{t} \operatorname{div}\left(\psi(t, \tau) \nabla y_{h}\right) d \tau \\
& \left.+\phi\left(y_{h}\right)-f-B u_{h}, e^{y}-e_{I}^{y}\right)
\end{aligned}
$$




$$
\begin{aligned}
+\sum_{\tau} \int_{\partial \tau} & {\left[\left(A \nabla y_{h}\right) \cdot n\right.} \\
& \left.\left.+\int_{0}^{t}\left(\psi(t, \tau) \nabla y_{h}(\tau)\right) \cdot n d \tau\right]\left(e^{y}-e_{I}^{y}\right)\right] .
\end{aligned}
$$

Then, we have

$$
\begin{aligned}
& \frac{1}{2} \frac{d}{d t}\left\|y_{h}-y\left(u_{h}\right)\right\|_{0, \Omega}^{2}+c\left\|y_{h}-y\left(u_{h}\right)\right\|_{1, \Omega}^{2} \\
& \leq \quad\left(\left(y_{h}-y\left(u_{h}\right)\right)_{t}, e^{y}\right)+a\left(y_{h}-y\left(u_{h}\right), e^{y}\right) \\
& \quad+\left(\phi\left(y_{h}\right)-\phi\left(y\left(u_{h}\right)\right), e^{y}\right) \\
& \leq \sum_{\tau} \int_{\tau}\left(y_{h t}-\operatorname{div}\left(A \nabla y_{h}\right)-\int_{0}^{t} \operatorname{div}\left(\psi(t, \tau) \nabla y_{h}\right) d \tau\right. \\
& \left.\quad+\phi\left(y_{h}\right)-f-B u_{h}, e^{y}-e_{I}^{y}\right) \\
& \quad+\sum_{\tau} \int_{\partial \tau}\left[\left(A \nabla y_{h}\right) \cdot n+\int_{0}^{t}\left(\psi(t, \tau) \nabla y_{h}(\tau)\right) \cdot n d \tau\right] \\
& \quad \times\left(e^{y}-e_{I}^{y}\right) \\
& \quad-\int_{0}^{t} \psi\left(t, \tau ;\left(y_{h}-y\left(u_{h}\right)\right)(\tau), e^{y}\right) d \tau .
\end{aligned}
$$

By integrating time from 0 to $t$ in the above inequality, combining (6) and the Schwartz inequality, we have

$$
\begin{aligned}
& \frac{1}{2}\left\|y_{h}-y\left(u_{h}\right)\right\|_{0, \Omega}^{2}+c \int_{0}^{t}\left\|y_{h}-y\left(u_{h}\right)\right\|_{1, \Omega}^{2} d \tau \\
& \leq C \int_{0}^{t} \sum_{\tau} h_{\tau}^{2} \int_{\tau}\left(y_{h t}-\operatorname{div}\left(A \nabla y_{h}\right)\right. \\
& \quad-\int_{0}^{t} \operatorname{div}\left(\psi(t, \tau) \nabla y_{h}\right) d \tau \\
& \left.+\phi\left(y_{h}\right)-f-B u_{h}\right)^{2} d \tau \\
& +\int_{0}^{t} \sum_{\tau} h_{l} \int_{\partial \tau}\left[\left(A \nabla y_{h}\right) \cdot n\right. \\
& \left.\left.+\delta \int_{0}^{t}\left\|y_{h}-y\left(u_{h}\right)\right\|_{1, \Omega}^{2} d \tau(t, \tau) \nabla y_{h}(\tau)\right) \cdot n d \tau\right]^{2} d \tau \\
& +C \int_{0}^{t} \int_{\tau}\left\|\left(y_{h}-y\left(u_{h}\right)\right)(s)\right\|_{1, \Omega}^{2} d s d \tau \\
& +\left\|y_{h}(x, 0)-y_{0}(x)\right\|_{L^{2}(\Omega)}^{2}
\end{aligned}
$$

Since $\delta$ is small enough, then from (49) and the Gronwall inequality, we have

$$
\begin{gathered}
\int_{0}^{t}\left\|y_{h}-y\left(u_{h}\right)\right\|_{1, \Omega}^{2} d \tau \\
\leq C \int_{0}^{t} \sum_{\tau} h_{\tau}^{2} \int_{\tau}\left(y_{h t}-\operatorname{div}\left(A \nabla y_{h}\right)\right. \\
-\int_{0}^{t} \operatorname{div}\left(\psi(t, \tau) \nabla y_{h}\right) d \tau \\
\left.+\phi\left(y_{h}\right)-f-B u_{h}\right)^{2} d \tau \\
+C \int_{0}^{t} \sum_{\tau} h_{l} \int_{\partial \tau}\left[\left(A \nabla y_{h}\right) \cdot n+\int_{0}^{t}\left(\psi(t, \tau) \nabla y_{h}(\tau)\right)\right. \\
\cdot n d \tau]^{2} d \tau+\left\|y_{h}(x, 0)-y_{0}(x)\right\|_{L^{2}(\Omega)}^{2}
\end{gathered}
$$

So, by using the inequality (50) we obtain

$$
\left\|y_{h}-y\left(u_{h}\right)\right\|_{L^{2}\left(J ; H^{1}(\Omega)\right)}^{2} \leq C \sum_{i=2}^{4} \eta_{i}^{2} .
$$

This completes the proof.

Analogous to Theorem 3, we can prove the following estimates.

Theorem 4. Let $\left(y\left(u_{h}\right), p\left(u_{h}\right)\right)$ and $\left(y_{h}, p_{h}\right)$ be the solutions of (23)-(26) and (18)-(22), respectively. Then

$$
\left\|p\left(u_{h}\right)-p_{h}\right\|_{L^{2}\left(J ; H^{1}(\Omega)\right)}^{2} \leq C \sum_{i=2}^{6} \eta_{i}^{2},
$$

where

$$
\begin{aligned}
\eta_{5}^{2}=\int_{0}^{T} \sum_{\tau \in \mathscr{T}^{h}} h_{\tau}^{2} \int_{\tau}( & y_{h}-y_{0}+p_{h t}+\operatorname{div}\left(A^{*} \nabla p_{h}\right) \\
& +\int_{t}^{T} \operatorname{div}\left(\psi^{*}(\tau, t) \nabla p_{h}(\tau)\right) d \tau \\
& \left.-\phi^{\prime}\left(y_{h}\right) p_{h}\right)^{2} d \tau d t \\
\eta_{6}^{2}=\int_{0}^{T} \sum_{\tau \in \mathscr{T}^{h}} h_{l} \int_{\partial \tau}\left[\left(A^{*} \nabla p_{h}\right) \cdot n\right. & \\
& \left.+\int_{0}^{t}\left(\left(\psi^{*}(t, \tau) \nabla p_{h}(\tau)\right) \cdot n\right) d \tau\right]^{2} d l d t
\end{aligned}
$$

where $\eta_{2}-\eta_{4}$ are defined in Theorem 3, $l$ is a face of an element $\tau$, and $\left[\left(A^{*} \nabla p_{h}\right) \cdot n\right]$ is the A-normal derivative jump over the interior face $l$, defined by

$$
\left[\left(A^{*} \nabla p_{h}\right) \cdot n\right]_{l}=\left(\left.A^{*} \nabla p_{h}\right|_{\tau_{l}^{1}}-\left.A^{*} \nabla p_{h}\right|_{\tau_{l}^{2}}\right) \cdot n,
$$


Journal of Applied Mathematics

7

where $n$ is the unit normal vector on $l=\bar{\tau}_{l}^{1} \cap \bar{\tau}_{l}^{2}$ outwards $\tau_{l}^{1}$. For convenience, one defines $\left[\left(A \nabla p_{h}\right) \cdot n\right]_{l}=0$ when $l \subset \partial \Omega$.

Proof. Let $e^{p}=p\left(u_{h}\right)-p_{h}$, and let $e_{I}^{p}=\widehat{\pi}_{h} e^{p}$, where $\widehat{\pi}_{h}$ is the Clément-type interpolator defined in Lemma 1. Then, from (20) and (25), we obtain

$$
\begin{aligned}
& -\left(q_{h},\left(p_{h}-p\left(u_{h}\right)\right)_{t}\right)+a\left(q_{h}, p_{h}-p\left(u_{h}\right)\right) \\
& \quad+\int_{t}^{T} \psi\left(\tau, t ; q_{h}(t),\left(p_{h}-p\left(u_{h}\right)\right)(\tau)\right) d \tau \\
& \quad+\left(\phi^{\prime}\left(y_{h}\right) p_{h}-\phi^{\prime}\left(y\left(u_{h}\right)\right) p\left(u_{h}\right), q_{h}\right) \\
& =\left(y_{h}-y\left(u_{h}\right), q_{h}\right), \quad \forall q_{h} \in V_{h} .
\end{aligned}
$$

Namely,

$$
\begin{aligned}
-\left(q_{h},\right. & \left.\left(p_{h}-p\left(u_{h}\right)\right)_{t}\right)+a\left(q_{h}, p_{h}-p\left(u_{h}\right)\right) \\
& +\int_{t}^{T} \psi\left(\tau, t ; q_{h}(t),\left(p_{h}-p\left(u_{h}\right)\right)(\tau)\right) d \tau \\
& +\left(\phi^{\prime}\left(y_{h}\right)\left(p_{h}-p\left(u_{h}\right)\right), q_{h}\right) \\
= & \left(y_{h}-y\left(u_{h}\right), q_{h}\right) \\
& -\left(\left(\phi^{\prime}\left(y_{h}\right)-\phi^{\prime}\left(y\left(u_{h}\right)\right)\right) p\left(u_{h}\right), q_{h}\right) .
\end{aligned}
$$

By using (56), we obtain

$$
\begin{aligned}
-\left(e^{p}\right. & \left.,\left(p_{h}-p\left(u_{h}\right)\right)_{t}\right)+a\left(e^{p}, p_{h}-p\left(u_{h}\right)\right) \\
& +\int_{t}^{T} \psi\left(\tau, t ; e^{p}(t),\left(p_{h}-p\left(u_{h}\right)\right)(\tau)\right) d \tau \\
& +\left(\phi^{\prime}\left(y_{h}\right)\left(p_{h}-p\left(u_{h}\right)\right), e^{p}\right) \\
= & -\left(e^{p}-e_{I}^{p},\left(p_{h}-p\left(u_{h}\right)\right)_{t}\right)+a\left(e^{p}-e_{I}^{p}, p_{h}-p\left(u_{h}\right)\right) \\
& +\int_{t}^{T} \psi\left(\tau, t ;\left(e^{p}-e_{I}^{p}\right)(t),\left(p_{h}-p\left(u_{h}\right)\right)(\tau)\right) d \tau \\
& +\left(\phi^{\prime}\left(y_{h}\right)\left(p_{h}-p\left(u_{h}\right)\right), e^{p}-e_{I}^{p}\right) \\
& -\left(e_{I}^{p},\left(p_{h}-p\left(u_{h}\right)\right)_{t}\right)+a\left(e_{I}^{p}, p_{h}-p\left(u_{h}\right)\right) \\
& +\int_{t}^{T} \psi\left(\tau, t ; e_{I}^{p}(t),\left(p_{h}-p\left(u_{h}\right)\right)(\tau)\right) d \tau \\
& +\left(\phi^{\prime}\left(y_{h}\right)\left(p_{h}-p\left(u_{h}\right)\right), e_{I}^{p}\right) \\
= & \left.-\left(e^{p}-e_{I}^{p},\left(p_{h}-p\left(u_{h}\right)\right)\right)_{t}\right)+a\left(e^{p}-e_{I}^{p}, p_{h}-p\left(u_{h}\right)\right) \\
& +\int_{t}^{T} \psi\left(\tau, t ;\left(e^{p}-e_{I}^{p}\right)(t),\left(p_{h}-p\left(u_{h}\right)\right)(\tau)\right) d \tau \\
+ & \left(\phi^{\prime}\left(y_{h}\right)\left(p_{h}-p\left(u_{h}\right)\right), e^{p}-e_{I}^{p}\right)+\left(y_{h}-y\left(u_{h}\right), e_{I}^{p}\right) \\
& \left.-\left(\phi^{\prime}\left(y\left(u_{h}\right)\right)\right) p\left(u_{h}\right), e_{I}^{p}\right)
\end{aligned}
$$

$$
\begin{aligned}
= & -\left(e^{p}-e_{I}^{p}, p_{h t}\right)+a\left(e^{p}-e_{I}^{p}, p_{h}\right) \\
& +\int_{t}^{T} \psi\left(\tau, t ;\left(e^{p}-e_{I}^{p}\right)(t), p_{h}\right)(\tau) d \tau \\
& +\left(\phi^{\prime}\left(y_{h}\right)\left(p_{h}\right), e^{p}-e_{I}^{p}\right) \\
& +\left(e^{p}-e_{I}^{p}, p_{t}\left(u_{h}\right)\right)-a\left(e^{p}-e_{I}^{p}, p\left(u_{h}\right)\right) \\
& -\int_{t}^{T} \psi\left(\tau, t ;\left(e^{p}-e_{I}^{p}\right)(t), p\left(u_{h}\right)(\tau)\right) d \tau \\
& -\left(\phi^{\prime}\left(y_{h}\right)\left(p\left(u_{h}\right)\right), e^{p}-e_{I}^{p}\right)+\left(y_{h}-y\left(u_{h}\right), e_{I}^{p}\right) \\
& -\left(\left(\phi^{\prime}\left(y_{h}\right)-\phi^{\prime}\left(y\left(u_{h}\right)\right)\right) p\left(u_{h}\right), e_{I}^{p}\right) \\
= & \sum_{\tau} \int_{\tau}\left(-y_{h}+y_{0}-p_{h t}-\operatorname{div}\left(A^{*} \nabla p_{h}\right)\right. \\
& \quad-\left(\left(\phi^{\prime}\left(y_{h}\right)-\phi^{\prime}\left(y\left(u_{h}\right)\right)\right) p\left(u_{h}\right), e^{p}\right) \cdot \\
& \times\left(e^{p}-e_{I}^{p}\right) \\
& +\sum_{\tau} \int_{\partial \tau}\left(\left(A^{*} \nabla p_{h}\right) \cdot n+\int_{0}^{t}\left(\left(\psi^{*}(t, \tau) \nabla p_{h}(\tau)\right) \cdot n\right) d \tau\right) \\
& \left.\times\left(e^{p}-e_{I}^{p}\right)+\left(y_{h}-y\left(u_{h}\right), e_{I}^{p}\right) d \tau+\phi^{\prime}\left(y_{h}\right) p_{h}\right) \\
& \left(y^{\prime}\right)
\end{aligned}
$$

Then, we have

$$
\begin{aligned}
& -\frac{1}{2} \frac{d}{d t}\left\|p_{h}-p\left(u_{h}\right)\right\|_{0, \Omega}^{2}+c\left\|p_{h}-p\left(u_{h}\right)\right\|_{1, \Omega}^{2} \\
& \leq-\left(e^{p},\left(p_{h}-p\left(u_{h}\right)\right)_{t}\right)+a\left(e^{p}, p_{h}-p\left(u_{h}\right)\right) \\
& +\left(\phi^{\prime}\left(y_{h}\right)\left(p_{h}-p\left(u_{h}\right)\right), e^{p}\right) \\
& \leq \sum_{\tau} \int_{\tau}\left(-y_{h}+y_{0}-p_{h t}-\operatorname{div}\left(A^{*} \nabla p_{h}\right)\right. \\
& \quad-\int_{t}^{T} \operatorname{div}\left(\psi^{*}(\tau, t) \nabla p_{h}(\tau)\right) d \tau \\
& \left.+\phi^{\prime}\left(y_{h}\right) p_{h}\right)\left(e^{p}-e_{I}^{p}\right) \\
& +\sum_{\tau} \int_{\partial \tau}\left(\left(A^{*} \nabla p_{h}\right) \cdot n\right. \\
& +\int_{t}^{T} \psi\left(\tau, t ; e^{p}(t),\left(p_{h}-p\left(u_{h}\right)\right)(\tau)\right) d \tau . \\
& +\left(\left(\phi^{\prime}\left(y_{h}^{T}\left(\left(\psi^{*}(t, \tau) \nabla p_{h}(\tau)\right) \cdot n\right) d \tau\right)\right.\right. \\
& \times\left(e^{p}-e_{I}^{p}\right)+\left(y_{h}-y\left(u_{h}\right), e_{I}^{p}\right)
\end{aligned}
$$


By integrating time from $t$ to $T$ in the above inequality, combining (6) and the Schwartz inequality, we have

$$
\begin{aligned}
& \frac{1}{2}\left\|p_{h}-p\left(u_{h}\right)\right\|_{0, \Omega}^{2}+c \int_{t}^{T}\left\|p_{h}-p\left(u_{h}\right)\right\|_{1, \Omega}^{2} d \tau \\
& \leq C \int_{t}^{T} \sum_{\tau} h_{\tau}^{2} \int_{\tau}\left(y_{h}-y_{0}+p_{h t}+\operatorname{div}\left(A^{*} \nabla p_{h}\right)\right. \\
& +\int_{t}^{T} \operatorname{div}\left(\psi^{*}(\tau, t) \nabla p_{h}(\tau)\right) d \tau \\
& \left.-\phi^{\prime}\left(y_{h}\right) p_{h}\right)^{2} d \tau \\
& +\int_{t}^{T} \sum_{\tau} h_{l} \int_{\partial \tau}\left[\left(A^{*} \nabla p_{h}\right) \cdot n\right. \\
& \left.+\int_{t}^{T}\left(\left(\psi^{*}(t, \tau) \nabla p_{h}(\tau)\right) \cdot n\right) d \tau\right]^{2} d \tau \\
& +\delta \int_{t}^{T}\left\|p_{h}-p\left(u_{h}\right)\right\|_{1, \Omega}^{2} d \tau \\
& +C \int_{t}^{T} \int_{\tau}\left\|\left(p_{h}-p\left(u_{h}\right)\right)(s)\right\|_{1, \Omega}^{2} d s d \tau \\
& +C \int_{t}^{T} \int_{\tau}\left\|y_{h}-y\left(u_{h}\right)\right\|_{0, \Omega}^{2} d \tau
\end{aligned}
$$

Since $\delta$ is small enough, then from (59) and the Gronwall inequality, we have

$$
\begin{aligned}
& \int_{t}^{T}\left\|p_{h}-p\left(u_{h}\right)\right\|_{1, \Omega}^{2} d \tau \\
& \leq C \int_{t}^{T} \sum_{\tau} h_{\tau}^{2} \int_{\tau}\left(y_{h}-y_{0}+p_{h t}+\operatorname{div}\left(A^{*} \nabla p_{h}\right)\right. \\
& +\int_{t}^{T} \operatorname{div}\left(\psi^{*}(\tau, t) \nabla p_{h}(\tau)\right) d \tau \\
& \left.-\phi^{\prime}\left(y_{h}\right) p_{h}\right)^{2} d \tau \\
& +\int_{t}^{T} \sum_{\tau} h_{l} \int_{\partial \tau}\left[\left(A^{*} \nabla p_{h}\right) \cdot n\right. \\
& \left.+\int_{t}^{T}\left(\left(\psi^{*}(t, \tau) \nabla p_{h}(\tau)\right) \cdot n\right) d \tau\right]^{2} d \tau \\
& +\left\|y\left(u_{h}\right)-y_{h}\right\|_{L^{2}\left(J ; L^{2}(\Omega)\right)}^{2}
\end{aligned}
$$

$$
\begin{gathered}
\leq C \int_{t}^{T} \sum_{\tau} h_{\tau}^{2} \int_{\tau}\left(y_{h}-y_{0}+p_{h t}+\operatorname{div}\left(A^{*} \nabla p_{h}\right)\right. \\
+\int_{t}^{T} \operatorname{div}\left(\psi^{*}(\tau, t) \nabla p_{h}(\tau)\right) d \tau \\
\left.-\phi^{\prime}\left(y_{h}\right) p_{h}\right)^{2} d \tau \\
+\int_{t}^{T} \sum_{\tau} h_{l} \int_{\partial \tau}\left[\left(A^{*} \nabla p_{h}\right) \cdot n\right. \\
\left.+\| \int_{t}^{T}\left(\left(\psi^{*}(t, \tau) \nabla p_{h}(\tau)\right) \cdot n\right) d \tau\right]^{2} d \tau \\
+\left\|y\left(u_{h}\right)-y_{h}\right\|_{L^{2}\left(J ; H^{1}(\Omega)\right.}^{2} .
\end{gathered}
$$

Finally, combine inequality (60) and Theorem 3 to obtain

$$
\left\|p\left(u_{h}\right)-p_{h}\right\|_{L^{2}\left(J ; H^{1}(\Omega)\right)}^{2} \leq C \sum_{i=2}^{6} \eta_{i}^{2} .
$$

This completes the proof.

Hence, we combine Theorems 2-4 to conclude.

Theorem 5. Let $(y, p, u)$ and $\left(y_{h}, p_{h}, u_{h}\right)$ be the solutions of (10)-(14) and (18)-(22), respectively. Then

$$
\begin{gathered}
\left\|u-u_{h}\right\|_{L^{2}\left(J ; L^{2}(\Omega)\right)}^{2}+\left\|y-y_{h}\right\|_{L^{2}\left(J ; H^{1}(\Omega)\right)}^{2} \\
+\left\|p-p_{h}\right\|_{L^{2}\left(J ; H^{1}(\Omega)\right)}^{2} \leq C \sum_{i=1}^{6} \eta_{i}^{2}
\end{gathered}
$$

where $\eta_{1}-\eta_{6}$ are defined in Theorems 2-4, respectively.

Proof. From (10)-(14) and (23)-(26), we obtain the error equations

$$
\begin{aligned}
& \left(y_{t}-y_{t}\left(u_{h}\right), w\right)+a\left(y-y\left(u_{h}\right), w\right) \\
& \quad+\int_{0}^{t} \psi\left(t, \tau ;\left(y-y\left(u_{h}\right)\right)(\tau), w_{h}\right) d \tau \\
& \quad+\left(\phi(y)-\phi\left(y\left(u_{h}\right)\right), w\right)=\left(B\left(u-u_{h}\right), w\right), \\
& -\left(p_{t}-p_{t}\left(u_{h}\right), q\right)+a\left(q, p-p\left(u_{h}\right)\right) \\
& \quad+\int_{t}^{T} \psi\left(\tau, t ; q_{h}(t),\left(p-p\left(u_{h}\right)\right)(\tau)\right) d \tau \\
& \quad+\left(\phi^{\prime}(y) p-\phi^{\prime}\left(y\left(u_{h}\right)\right) p\left(u_{h}\right), q\right)=\left(y-y\left(u_{h}\right), q\right)
\end{aligned}
$$

for all $w \in V$ and $q \in V$. Thus, it follows from (63) that

$$
\begin{aligned}
& \left(y_{t}-y_{t}\left(u_{h}\right), w\right)+a\left(y-y\left(u_{h}\right), w\right) \\
& \quad+\int_{0}^{t} \psi\left(t, \tau ;\left(y-y\left(u_{h}\right)\right)(\tau), w_{h}\right) d \tau \\
& \quad+\left(\phi(y)-\phi\left(y\left(u_{h}\right)\right), w\right)=\left(B\left(u-u_{h}\right), w\right),
\end{aligned}
$$




$$
\begin{aligned}
& -\left(p_{t}-p_{t}\left(u_{h}\right), q\right)+a\left(q, p-p\left(u_{h}\right)\right) \\
& \quad+\int_{t}^{T} \psi\left(\tau, t ; q_{h}(t),\left(p-p\left(u_{h}\right)\right)(\tau)\right) d \tau \\
& \quad+\left(\phi^{\prime}\left(y\left(u_{h}\right)\right)\left(p-p\left(u_{h}\right)\right), q\right) \\
& =\left(y-y\left(u_{h}\right), q\right)+\left(\widetilde{\phi}^{\prime \prime}\left(y\left(u_{h}\right)\right)\left(y\left(u_{h}\right)-y\right) p, q\right) .
\end{aligned}
$$

By using the stability results in $[17,19]$, then we can prove that

$$
\begin{aligned}
\left\|y-y\left(u_{h}\right)\right\|_{L^{2}\left(J ; H^{1}(\Omega)\right)}^{2} & \leq C\left\|u-u_{h}\right\|_{L^{2}\left(J ; L^{2}(\Omega)\right)}^{2}, \\
\left\|p-p\left(u_{h}\right)\right\|_{L^{2}\left(J ; H^{1}(\Omega)\right)}^{2} & \leq\left\|y-y\left(u_{h}\right)\right\|_{L^{2}\left(J ; H^{1}(\Omega)\right)}^{2} \\
& \leq C\left\|u-u_{h}\right\|_{L^{2}\left(J ; L^{2}(\Omega)\right)}^{2} .
\end{aligned}
$$

Finally, combining Theorems 2-4 and (65) leads to (62).

\section{An Adaptive Algorithm}

In this section, we introduce an adaptive algorithm to guide the mesh refinement process. A posteriori error estimates which have been derived in Section 3 are used as an error indicator to guide the mesh refinement in adaptive finite element method.

Now, we discuss the adaptive mesh refinement strategy. The general idea is to refine the mesh such that the error indicator like $\eta$ is equally distributed over the computational mesh. Assume that an a posteriori error estimator $\eta$ has the form of $\eta^{2}=\sum_{\tau_{i}} \eta_{\tau_{i}}^{2}$, where $\tau_{i}$ is the finite elements. At each iteration, an average quantity of all $\eta_{\tau_{i}}^{2}$ is calculated, and each $\eta_{\tau_{i}}^{2}$ is then compared with this quantity. The element $\tau_{i}$ is to be refined if $\eta_{\tau_{i}}^{2}$ is larger than this quantity. As $\eta_{\tau_{i}}^{2}$ represents the total approximation error over $\tau_{i}$, this strategy makes sure that higher density of nodes is distributed over the area where the error is higher.

Based on this principle, we define an adaptive algorithm of the semilinear parabolic integrodifferential optimal control problems (1)-(2) as follows: starting from initial triangulations $\mathscr{T}_{h_{0}}$ of $\Omega$, we construct a sequence of refined triangulation $\mathscr{T}_{h_{j}}$ as follows. Given $\mathscr{T}_{h_{j}}$, we compute the solutions $\left(y_{h}, p_{h}, u_{h}\right)$ of the system (18)-(22) and their error estimator as follows:

$$
\begin{gathered}
\eta_{\tau}^{2}=\int_{0}^{T} \sum_{\tau \in \mathscr{T}_{h}} h_{\tau}^{1+s}\left\|\alpha u_{h}+B^{*} p_{h}\right\|_{H^{s}(\tau)}^{1+s} d t \\
+\int_{0}^{T} \sum_{\tau \in \mathscr{T}^{h}} h_{\tau}^{2} \int_{\tau}\left(f+B u_{h}-y_{h t}+\operatorname{div}\left(A \nabla y_{h}\right)\right. \\
+\int_{0}^{t} \operatorname{div}\left(\psi(t, \tau) \nabla y_{h}(\tau)\right) d \tau \\
\left.-\phi\left(y_{h}\right)\right)^{2} d \tau d t
\end{gathered}
$$

$$
\begin{aligned}
& +\int_{0}^{T} \sum_{\tau \in \mathscr{T}^{h}} h_{l} \int_{\partial \tau}\left[\left(A \nabla y_{h}\right) \cdot n\right. \\
& \left.+\int_{0}^{t}\left(\left(\psi(t, \tau) \nabla y_{h}(\tau)\right) \cdot n\right) d \tau\right]^{2} d l d t \\
& +\left\|y_{h}(x, 0)-y_{0}(x)\right\|_{L^{2}(\Omega)}^{2} \\
& +\int_{0}^{T} \sum_{\tau \in \mathscr{T}^{h}} h_{\tau}^{2} \int_{\tau}\left(y_{h}-y_{0}+p_{h t}+\operatorname{div}\left(A^{*} \nabla p_{h}\right)\right. \\
& +\int_{t}^{T} \operatorname{div}\left(\psi^{*}(\tau, t) \nabla p_{h}(\tau)\right) d \tau \\
& \left.-\phi^{\prime}\left(y_{h}\right) p_{h}\right) d \tau d t \\
& +\int_{0}^{T} \sum_{\tau \in \mathscr{T}^{h}} h_{l} \int_{\partial \tau}\left[\left(A^{*} \nabla p_{h}\right) \cdot n\right. \\
& +\int_{0}^{t}\left(\left(\psi^{*}(t, \tau) \nabla p_{h}(\tau)\right)\right. \\
& \cdot n) d \tau]^{2} d l d t \\
& E_{j}=\sum_{\tau \in \mathscr{T}_{h}} \eta_{\tau}^{2}
\end{aligned}
$$

Then, we adopt the following mesh refinement strategy: all the triangles $\tau \in \mathscr{T}_{h_{j}}$ satisfying $\eta_{\tau}^{2} \geq \rho E_{j} / n$ are divided into four new triangles in $\mathscr{T}_{h_{j+1}}$ by joining the midpoints of the edges, where $n$ is the number of the elements of $\mathscr{T}_{h_{j}}$ and $\rho$ is a given constant. In order to maintain the new triangulation $\mathscr{T}_{h_{j+1}}$ to be regular and conformal, some additional triangles need to be divided into two or four new triangles depending on whether they have one or more neighbors which have been refined. Then, we obtain the new mesh $\mathscr{T}_{h_{j+1}}$. The above procedure will continue until $E_{j} \leq$ tol, where tol is a given tolerance error.

\section{Numerical Example}

In this section, we will give a numerical example to illustrate our theoretical results. Our numerical example is the following semilinear parabolic integrodifferential optimal control problem:

$$
\begin{aligned}
& \min _{u(t) \in K}\left\{\int_{0}^{1}\left(\frac{1}{2}\left\|y-y_{0}\right\|^{2}+\frac{1}{2}\left\|u-u_{0}\right\|^{2}\right) d t\right\} \\
& y_{t}-\operatorname{div}(\nabla y(x, t))-\int_{0}^{t} \operatorname{div}(\psi(t, \tau) \nabla y(x, \tau)) d \tau \\
& +\phi(y)=f+u, \quad x \in \Omega, t \in J,
\end{aligned}
$$




$$
\begin{gathered}
y(x, t)=0, \quad x \in \partial \Omega, t \in J, \\
y(x, 0)=0, \quad x \in \Omega, \\
-p_{t}-\operatorname{div}(\nabla p(x, t))-\int_{t}^{1} \operatorname{div}\left(\psi^{*}(\tau, t) \nabla p(x, \tau)\right) d \tau \\
+\phi^{\prime}(y) p=y-y_{0}, \quad x \in \Omega, t \in J, \\
p(x, t)=0, \quad x \in \partial \Omega, t \in J, \\
p(x, 1)=0, \quad x \in \Omega .
\end{gathered}
$$

In this example, we choose the domain $\Omega=[0,1] \times[0,1]$. Let $\Omega$ be partitioned into $\mathscr{T}_{h}$ as described Section 2 . For the constrained optimization problem:

$$
\min _{u \in K \subset U} \int_{0}^{1} S(u) d t,
$$

where $S(u)=(1 / 2)\left\|y-y_{0}\right\|^{2}+(1 / 2)\left\|u-u_{0}\right\|^{2}$ is a convex functional on $U$ and $K=\{u \in U: u \geq 0$ a.e. in $\Omega \times J\}$; the iterative scheme reads $(n=0,1,2, \ldots)$

$$
\begin{gathered}
b\left(u_{n+1 / 2}, v\right)=b\left(u_{n}, v\right)-\rho_{n}\left(S^{\prime}\left(u_{n}\right), v\right), \quad \forall v \in U, \\
u_{n+1}=P_{K}^{b}\left(u_{n+1 / 2}\right),
\end{gathered}
$$

where $b(\cdot, \cdot)$ is a symmetric and positive definite bilinear form such that there exist constants $c_{0}$ and $c_{1}$ satisfying

$$
\begin{gathered}
|b(u, v)| \leq c_{1}\|u\|_{U}\|v\|_{U}, \quad \forall u, v \in U, \\
b(u, u) \geq c_{0}\|u\|_{U}^{2},
\end{gathered}
$$

and the projection operator $P_{K}^{b} U \rightarrow K$ is defined: for given $w \in U$ find $P_{K}^{b} w \in K$ such that

$$
b\left(P_{K}^{b} w-w, P_{K}^{b} w-w\right)=\min _{u \in K} b(u-w, u-w) .
$$

The bilinear form $b(\cdot, \cdot)$ provides suitable preconditioning for the projection algorithm. An application of (69) to the discretized semilinear parabolic integrodifferential optimal control problem yields the following algorithm:

$$
\begin{gathered}
b\left(u_{n+1 / 2}, v_{h}\right)=b\left(u_{n}, v_{h}\right)-\rho_{n}\left(u_{n}+p_{n}, v_{h}\right), \quad \forall v_{h} \in K_{h} \\
\int_{0}^{1}\left(\left(y_{t}, w\right)+a(y, w)+\int_{0}^{t} \psi(t, \tau ; y(\tau), w) d \tau\right. \\
\quad+(\phi(y), w)) d t=\int_{0}^{1}(f+u, w) d t, \quad \forall w \in V, \\
\int_{0}^{1}\left(-\left(p_{t}, q\right)+a(q, p)+\int_{t}^{1} \psi(\tau, t ; q, p(\tau)) d \tau\right. \\
\left.+\left(\phi^{\prime}(y) p, q\right)\right) d t=\int_{0}^{1}\left(y-y_{0}, q\right) d t, \quad \forall q \in V, \\
u_{n+1}=P_{K}^{b}\left(u_{n+1 / 2}\right), \quad u_{n+1 / 2}, u_{n} \in K_{h} .
\end{gathered}
$$

The main computational effort is to solve the state and costate equations and to compute the projection $P_{K}^{b} u_{n+1 / 2}$. In this paper, we use a fast algebraic multigrid solver to solve the state and costate equations. Then, it is clear that the key to saving computing time is how to compute $P_{K}^{b} u_{n+1 / 2}$ efficiently. For the piecewise constant elements, $K_{h}=\left\{u_{h}: u_{h} \geq 0\right\}$ and $b(u, v)=(u, v)_{U}$; then

$$
\left.P_{K}^{b} u_{n+1 / 2}\right|_{\tau}=\max \left(0,\left.\operatorname{avg}\left(u_{n+1 / 2}\right)\right|_{\tau}\right),
$$

where $\left.\operatorname{avg}\left(u_{n+1 / 2}\right)\right|_{\tau}$ is the average of $u_{n+1 / 2}$ over $\tau$.

In solving our discretized optimal control problem, we use the preconditioned projection gradient method with $b(u, v)=(u, v)_{U}$ and a fixed step size $\rho=0.9$. We now briefly describe the solution algorithm to be used for solving the numerical example in this section as follows.

(1) Solve the discretized optimization problem with the projection gradient method on the current meshes and calculate the error estimators $\eta_{i}$.

(2) Adjust the meshes using the estimators and update the solution on new meshes, as described.

Now, we give a numerical example to illustrate our theoretical results.

Example 1. Let $\psi(t, \tau)=1, \phi(y)=y^{5}$. We choose the state function by

$$
y\left(x_{1}, x_{2}\right)=2 \sin \pi x_{1} \sin \pi x_{2} \sin \pi t .
$$

The function $f$ is given by $f(x)=y_{t}-\operatorname{div}(\nabla y(x, t))-$ $\int_{0}^{t} \operatorname{div}(\nabla y(x, \tau)) d \tau+y^{5}-u$. The costate function can be chosen as

$$
p\left(x_{1}, x_{2}\right)=\sin \pi x_{1} \sin \pi x_{2} \sin \pi t .
$$

The function $y_{0}$ is given by $y_{0}(x)=y+p_{t}+\operatorname{div}(\nabla p(x, t))+$ $\int_{t}^{1} \operatorname{div}(\nabla p(x, \tau)) d \tau-5 y^{4} p$. We assume that

$$
\begin{gathered}
\lambda= \begin{cases}0.8, & x_{1}+x_{2}>1.0 \\
0.3, & x_{1}+x_{2} \leq 1.0\end{cases} \\
u_{0}\left(x_{1}, x_{2}\right)=1-\sin \frac{\pi x_{1}}{2}-\sin \frac{\pi x_{2}}{2}+\lambda .
\end{gathered}
$$

Thus, the control function is given by

$$
u\left(x_{1}, x_{2}\right)=\max \left(u_{0}-p, 0\right) .
$$

In this example, the control function $u$ has a strong discontinuity introduced by $u_{0}$. The control function $u$ is discretized by piecewise constant functions, whereas the state $y$ and the costate $p$ were approximated by piecewise linear functions. In Table 1 , numerical results of $u, y$, and $p$ on uniform and adaptive meshes are presented. It can be found that the adaptive meshes generated using our error indicators can save substantial computational work, in comparison with the uniform meshes. On the other hand, for the discontinuous control variable $u$, the accuracy has become better from the uniform meshes to the adaptive meshes in Table 1. 
TABLE 1: Numerical results on uniform and adaptive meshes.

\begin{tabular}{|c|c|c|c|c|c|c|}
\hline & \multicolumn{3}{|c|}{ On uniform mesh } & \multicolumn{3}{|c|}{ On adaptive mesh } \\
\hline & u & $y$ & $p$ & $u$ & $y$ & $p$ \\
\hline Nodes & 8097 & 8097 & 8097 & 1102 & 1969 & 1969 \\
\hline Sides & 23968 & 23968 & 23968 & 3143 & 5744 & 5744 \\
\hline Elements & 15872 & 15872 & 15872 & 2042 & 3776 & 3776 \\
\hline Dofs & 15872 & 15872 & 15872 & 2042 & 3776 & 3776 \\
\hline Total $L^{2}$ error & $4.312 e-03$ & $5.457 e-3$ & $2.869 e-3$ & $4.018 e-03$ & $5.365 e-3$ & $2.768 e-3$ \\
\hline
\end{tabular}

\section{Conclusion and Future Works}

In this paper, we discuss the semi-discrete finite element methods of the semilinear parabolic integrodifferential optimal control problems (1)-(2). We have established a posteriori error estimates for each the state, the costate, and the control approximation. The posteriori error estimates for those problems by finite element methods seem to be new.

In our future work, we will use the mixed finite element method to deal with nonlinear parabolic integrodifferential optimal control problems. Furthermore, we will consider a posteriori error estimates and superconvergence of mixed finite element solution for nonlinear parabolic integrodifferential optimal control problems.

\section{Acknowledgments}

The author expresses his thanks to the referees for their helpful suggestions, which led to improvements of the presentation. This work is supported by the National Science Foundation of China (11201510), Mathematics TianYuan Special Funds of the National Natural Science Foundation of China (11126329), China Postdoctoral Science Foundation funded project (2011M500968), Natural Science Foundation Project of CQ CSTC (cstc2012jjA00003), Natural Science Foundation of Chongqing Municipal Education Commission (KJ121113), and Science and Technology Project of Wanzhou District of Chongqing (2013030050).

\section{References}

[1] R. S. Falk, "Approximation of a class of optimal control problems with order of convergence estimates," Journal of Mathematical Analysis and Applications, vol. 44, pp. 28-47, 1973.

[2] T. Geveci, "On the approximation of the solution of an optimal control problem governed by an elliptic equation," RAIRO Analyse Numérique, vol. 13, no. 4, pp. 313-328, 1979.

[3] E. Casas, M. Mateos, and F. Tröltzsch, "Error estimates for the numerical approximation of boundary semilinear elliptic control problems," Computational Optimization and Applications, vol. 31, no. 2, pp. 193-219, 2005.

[4] Z. Lu, "A posteriori error estimates of finite element methods for nonlinear quadratic boundary optimal control problem," Numerical Analysis and Applications, vol. 4, pp. 210-222, 2011.

[5] Y. Chen and W. Liu, "A posteriori error estimates for mixed finite element solutions of convex optimal control problems," Journal of Computational and Applied Mathematics, vol. 211, no. 1, pp. 76-89, 2008.
[6] D. A. French and J. T. King, "Approximation of an elliptic control problem by the finite element method," Numerical Functional Analysis and Optimization, vol. 12, no. 3-4, pp. 299-314, 1991.

[7] M. D. Gunzburger and L. S. Hou, "Finite-dimensional approximation of a class of constrained nonlinear optimal control problems," SIAM Journal on Control and Optimization, vol. 34, no. 3, pp. 1001-1043, 1996.

[8] P. Neittaanmäki and D. Tiba, Optimal Control of Nonlinear Parabolic Systems: Theory, Algorithms and Applications, Marcel Dekker, New York, NY, USA, 1994.

[9] J.-L. Lions, Optimal Control of Systems Governed by Partial Differential Equations, Springer, Berlin, Germany, 1971.

[10] H. Brunner and N. Yan, "Finite element methods for optimal control problems governed by integral equations and integrodifferential equations," Applied Numerical Mathematics, vol. 47, pp. 173-187, 2003.

[11] W. Liu and N. Yan, "A posteriori error estimates for distributed convex optimal control problems," Numerische Mathematik, vol. 101, pp. 1-27, 2005.

[12] R. Verfürth, "A posteriori error estimates for nonlinear problems. Finite element discretizations of elliptic equations," Mathematics of Computation, vol. 62, no. 206, pp. 445-475, 1994.

[13] Y. Chen and Z. Lu, "Error estimates of fully discrete mixed finite element methods for semilinear quadratic parabolic optimal control problem," Computer Methods in Applied Mechanics and Engineering, vol. 199, no. 23-24, pp. 1415-1423, 2010.

[14] Y. Chen and Z. Lu, "Error estimates for parabolic optimal control problem by fully discrete mixed finite element methods," Finite Elements in Analysis and Design, vol. 46, no. 11, pp. 957965, 2010.

[15] Z. Lu and Y. Chen, "A posteriori error estimates of triangular mixed finite element methods for semilinear optimal control problems," Advances in Applied Mathematics and Mechanics, vol. 1, no. 2, pp. 242-256, 2009.

[16] Z. Lu and Y. Chen, " $L^{\infty}$-error estimates of triangular mixed finite element methods for optimal control problem govern by semilinear elliptic equation," Numerical Analysis and Applications, vol. 2, pp. 74-86, 2009.

[17] E. G. Yanik and G. Fairweather, "Finite element methods for parabolic and hyperbolic partial integro-differential equations," Nonlinear Analysis. Theory, Methods \& Applications, vol. 12, no. 8, pp. 785-809, 1988.

[18] W. Liu and N. Yan, "A posteriori error estimates for distributed convex optimal control problems," Advances in Computational Mathematics, vol. 15, no. 1-4, pp. 285-309, 2001.

[19] I. H. Sloan and V. Thomée, "Time discretization of an integrodifferential equation of parabolic type," SIAM Journal on Numerical Analysis, vol. 23, no. 5, pp. 1052-1061, 1986. 


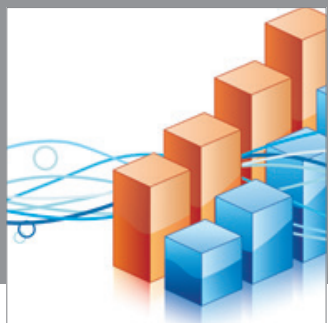

Advances in

Operations Research

mansans

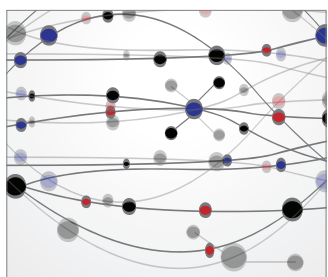

The Scientific World Journal
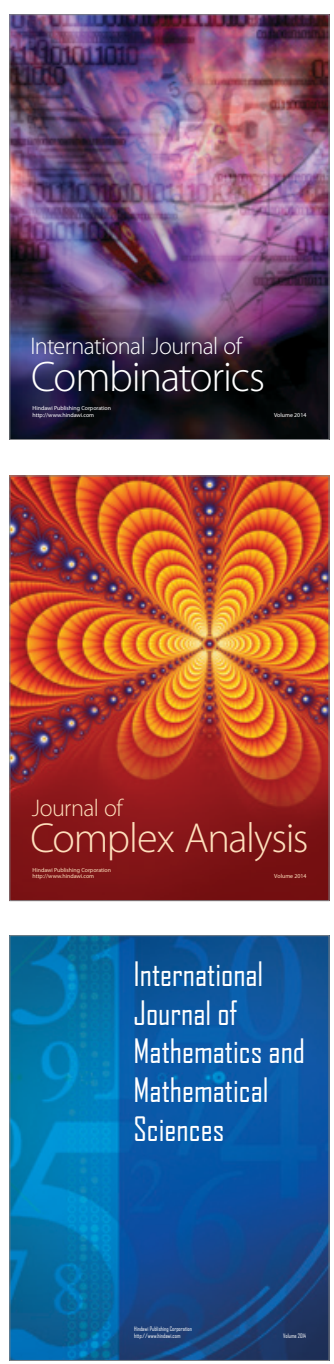
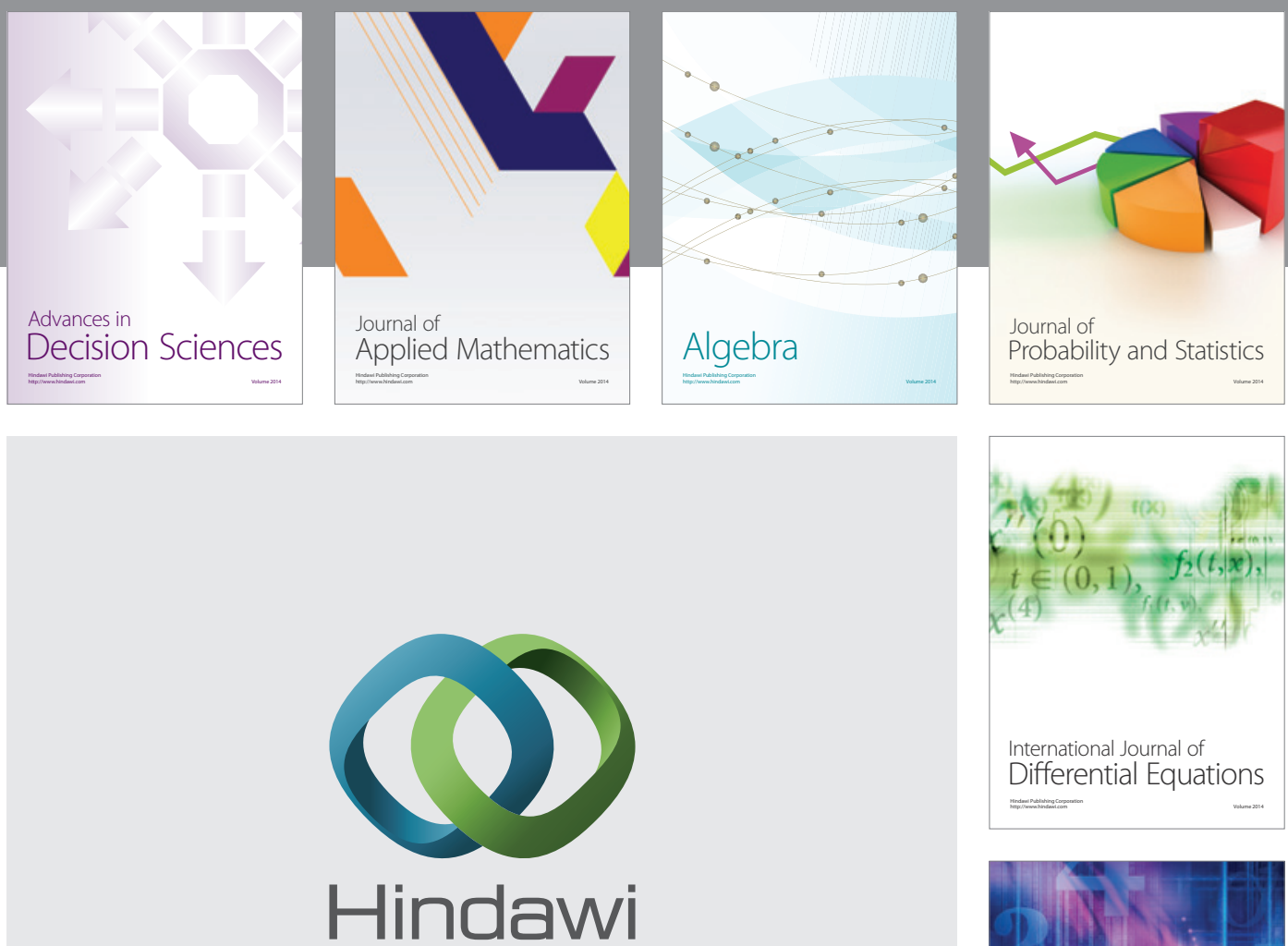

Submit your manuscripts at http://www.hindawi.com
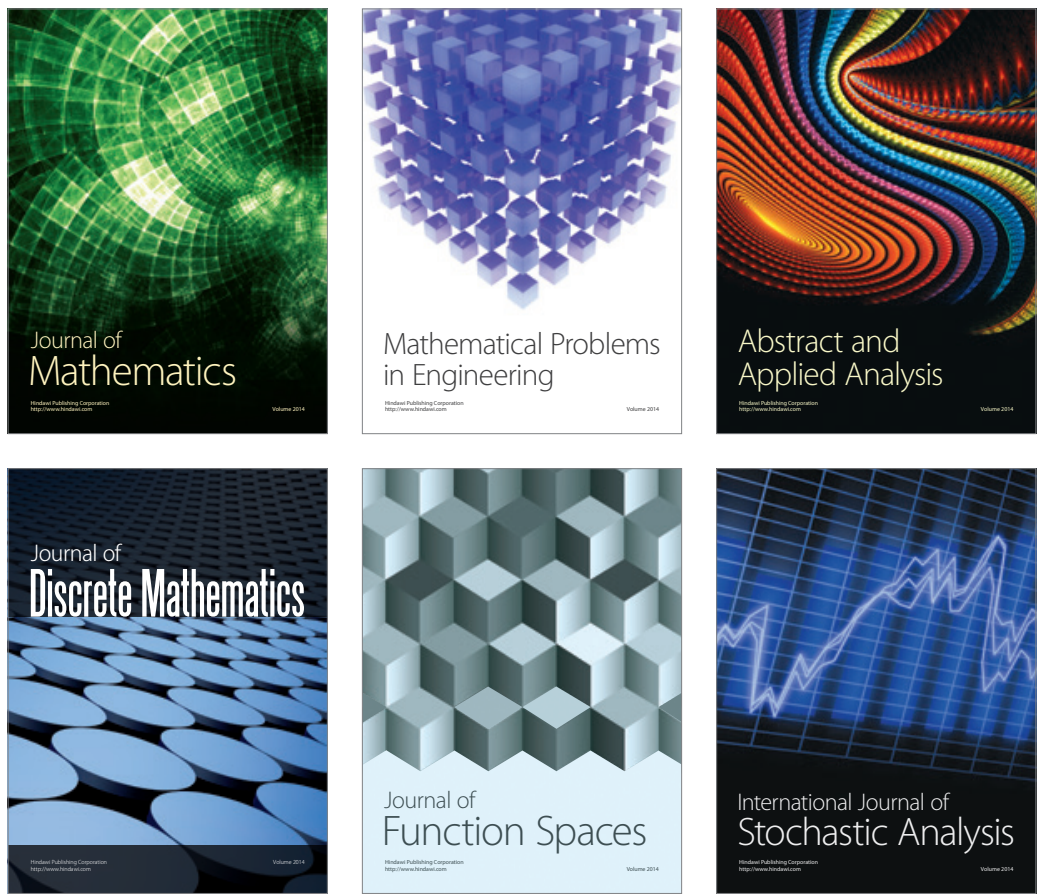

Journal of

Function Spaces

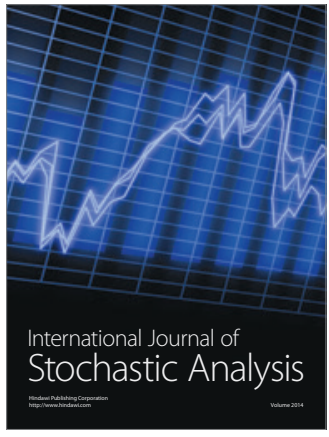

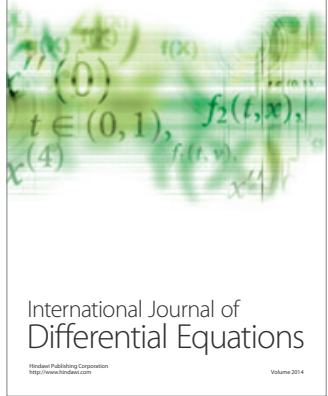
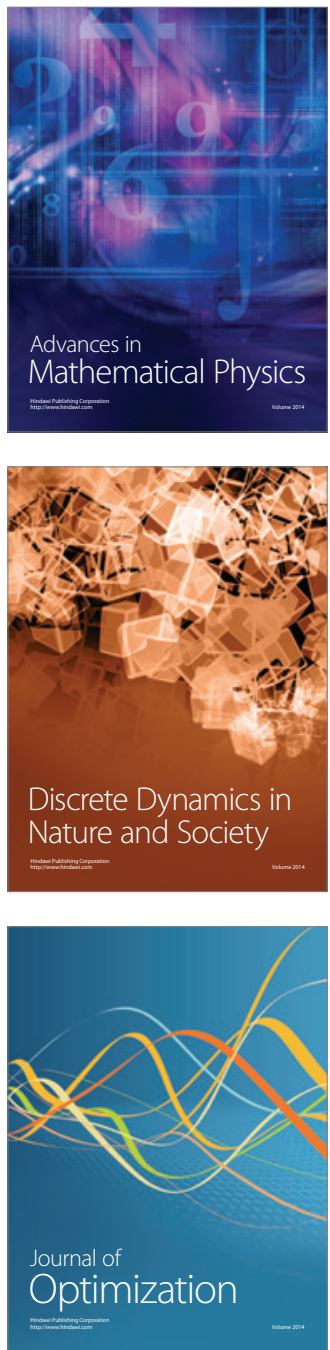